ISSN:1991-8178
EISSN: $2309-8414$
DOI: $10.22587 /$ ajbas.2017.11.16.4
Journal home page: www.ajbasweb.com

\title{
Embed Watermark Computing Using Logistic Map Chaotic System For Securing Medical Images
}

Rania Salah El-Sayed

Department of Mathematics \&Computer science, Faculty of Science, Al-Azhar University, Cairo. Egypt.

\section{Address For Correspondence:}

Rania Salah El-Sayed. Al-Azhar University, Faculty of Science,, Cairo. Egypt

E-mail: Rania5salah@yahoo.com

\section{A RTICLE INFO}

Article history:

Received 12 October 2017

Accepted 22 December 2017

Available online 31 December 2017

Keywords:

Chaotic map, logistic map, Region Of Interest (ROI), Region Of Non Interest (RONI), encryption, watermark.

\begin{abstract}
A B S T R A C T
Background: Securing medical image and patient's information while transfer or exchange these images remotely through hospital network provide image integrity and patient privacy. The concept of ROI (Region Of Interest) and RONI (Region Of Non Interest) are applied because the small bit of distortion in region of interest may lead to undesirable treatment for patient. Objective: We proposed to create watermark using logistic map chaotic system for encryption region of interest and embedded with patient's information into region of non interest of medical image. Results: After we applied our contributions by using multilayer security that combine Logistic map and watermark. And a variety of analyses and tests has been carried out on digital images such as: nuclear medicine, ultrasound, X-ray, computed radiography (CR) computed tomography (CT)...etc. The average of MSE equal about 0.4248 indicates that the MSE has very low consistent value compared to other information hiding methods of medical images. Conclusion: The experimental results prove that the proposed system can provide system authentication, image integrity and patient information confidentiality with high efficiency in health information system and ability of hide and secure patient's information without affect on quality of the original images. Performance analysis is observed using quality measure of reversed image in terms of Peak Signal to Noise Ratio (PSNR) and Mean Square Error (MSE).
\end{abstract}

INTRODUCTION

Tele-diagnosis (Musheer, Tanvir., 2012) includes transferring, sharing and distribution of patient's information and digital medical images over network raises the protect and secure digital magnetic resonance imaging (MRI), computed tomography (CT) scan, X-ray angiography, etc images from unauthorized access, modification and usage. Most of the security systems rely on the use of conventional encryption schemes like AES, DES etc. Currently conventional encryption schemes ( Musheer, Tanvir., 2012) aren't enough to provide sufficient security for image because of its property of bulky data and strong correlations among adjacent pixels, the chaotic systems have been used in modern cryptosystem. Chaos ( Quist A, Laurent $\mathrm{N}$, et al., 2015)has many features such as unpredictability, long periodicity, high sensitivity to initial condition parameter and mixing property, etc. These features are very important in cryptography.

In (Yong Wang, Kwok, 2009) proposed to use a chaos-based image encryption algorithm with variable control parameters. And using three maps in the permutation stage the Standard map, the Cat map or the Baker map achieve high performances against differential and statistical attacks.

In (Wei, Gouenou et al., 2010) This paper focus on the verification of medical image integrity through the combination of digital signatures with such a technology, and especially with Reversible Watermarking (RW).

In (Michael, Thomas et al., 2012) proposed to create encryption image Algorithm based on bit permutations and using an iterative process combined with a chaotic function. Their work achieved required

Open Access Journal

Published BY AENSI Publication

(C) 2017 AENSI Publisher All rights reserved

This work is licensed under the Creative Commons Attribution International License (CC BY).

http://creativecommons.org/licenses/by/4.0/

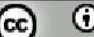


security level under many rounds of diffusion and confusion processes, but resulting in longer processing time and heavy computational load.

(Quist, Laurent et al.,2015) used encryption process is symmetric and uses client's authentication system to grant access but uses patients' unique information in encryption and watermarking of medical image.

(Yavuz, Erdem et al., 2015) used A chaos-based image encryption algorithm with simple logical functions. Using two independent chaotic functions one for low entropy plain images, which maintain their properties throughout many encryption rounds, a second chaotic function is incorporated to generate random numbers exploited together with exclusive-or operations for perturbing the integrity of such images even in first round. To increase the resistance of the crypto-system to differential attacks .

(Mamta Jain and Anil Kumar, 2017) used chaotic standard map, linear feedback shift register, and Rabin cryptosystem. And apply proposed algorithm on Brain disease stego and cover images.

To raising security and protection of MI and patient information in this paper we using the features of logistic map chaotic system to encrypt the significant part ROI of medical image and then embedded with patient information to RONI using concept of digital watermarking.

This paper is organized as follows. Section 1 is this introduction. Section 2 discusses preliminaries; Logistic map will be discussed as type of chaotic maps. We illustrate subsection as summary and other subsection as details of proposed system in methodology section 3. Section 4, provides the details of the conducted experiments along with results. The paper concludes with a brief summary of results and proposal of future research directions in section 5 .

\section{Preliminaries:}

This section provides a brief overview of Logistic map.

\subsection{Logistic map:}

Chaos theory (Hao Z, Xing-yuan W, Si-wei W, Kang G, Xiao-hui L.,2017), (Michael François, Thomas Grosges al., 2012) describes the behavior of certain nonlinear dynamic system that under specific conditions exhibit dynamics that are sensitive to initial conditions. The two basic properties of chaotic systems are the sensitivity to initial conditions and Mixing Property. In this paper, chaotic map is used to produce the chaotic sequence and used to control the encryption process. We proposed to use one of this maps named Logistic map (Hussain I, Shah T., 2013)

The Logistic map is one of the distinguished 1D chaotic maps. It is a simple dynamical equation with complex chaotic behavior namely better diffusion operation (Musheer, Tanvir., 2012). The mathematical definition of this map can be expressed in the equation (1).

$$
x_{n+1}=\mu x_{n}\left(1-x_{n}\right)
$$

Where $\boldsymbol{x}_{\boldsymbol{n}} \epsilon$ ]0,1[ that represent the ratio of existing population to the maximum possible population. The values of interest for the parameter $\boldsymbol{\mu}$ are those in the interval [0,4] and a property of the logistic map for most values of $\boldsymbol{\mu}$ between about 3.57 and 4 . Bifurcation diagram Fig (1) is an example for logistic map. In this example the bifurcation parameter $\boldsymbol{\mu}$ is the horizontal axis of the plot and the vertical axis is the set of values of the logistic function $\boldsymbol{x}_{\boldsymbol{n}}$ visited asymptotically from almost all initial conditions.

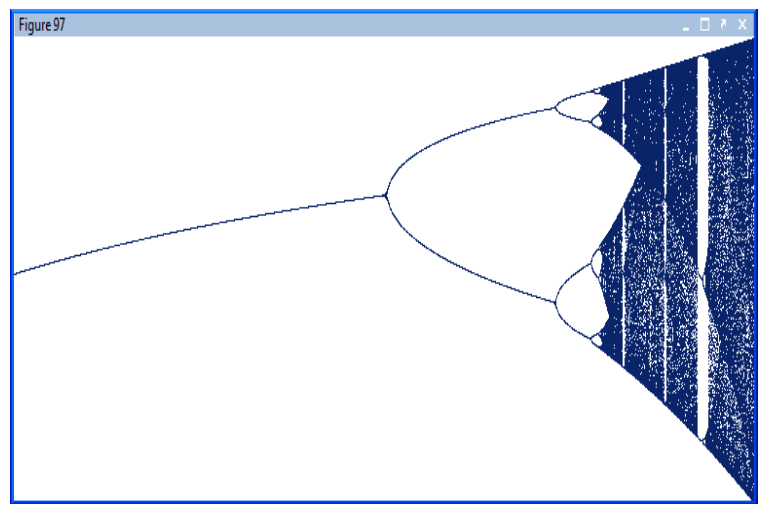

Fig. 1: Bifurcation diagram of the Logistic Map. 


\section{Methodology:}

Health sector concern about privacy, safety and security of medical image. Providing data authentication and integrity for medical image is very important. So we proposed medical image security system by fully recoverable and reversible technique for security medical image.

The summary of proposed framework is five phases to secure medical image for sender:

Phase.1: Extracting ROI from original image

Phase.2: Encrypt ROI based on logistic map

Phase.3: Convert cipher image to binary

Phase.4: Convert patient's information file from text document into binary.

Phase.5: Embedding watermarks based on 4level lifting Discrete Wavelet Transform (L-DWT) into RONI.

The summery of reverse framework to recover the medical image has six phases for receiver:

Phase.1: Extracting ROI from watermarked image depend on coordinate.

Phase.2: Specify the location of watermark by key-based detection.

Phase.3: Extract the watermarks (patient information \& binary cipher image)

Phase.4: Decrypt cipher image

Phase.5: Compare extracted ROI from phase 4 with the original one.

Phase.6: Combine ROI phase 1 and pre-output image after phase 3 to obtain original image.

The system diagram for the proposed approach is shown Fig (2).

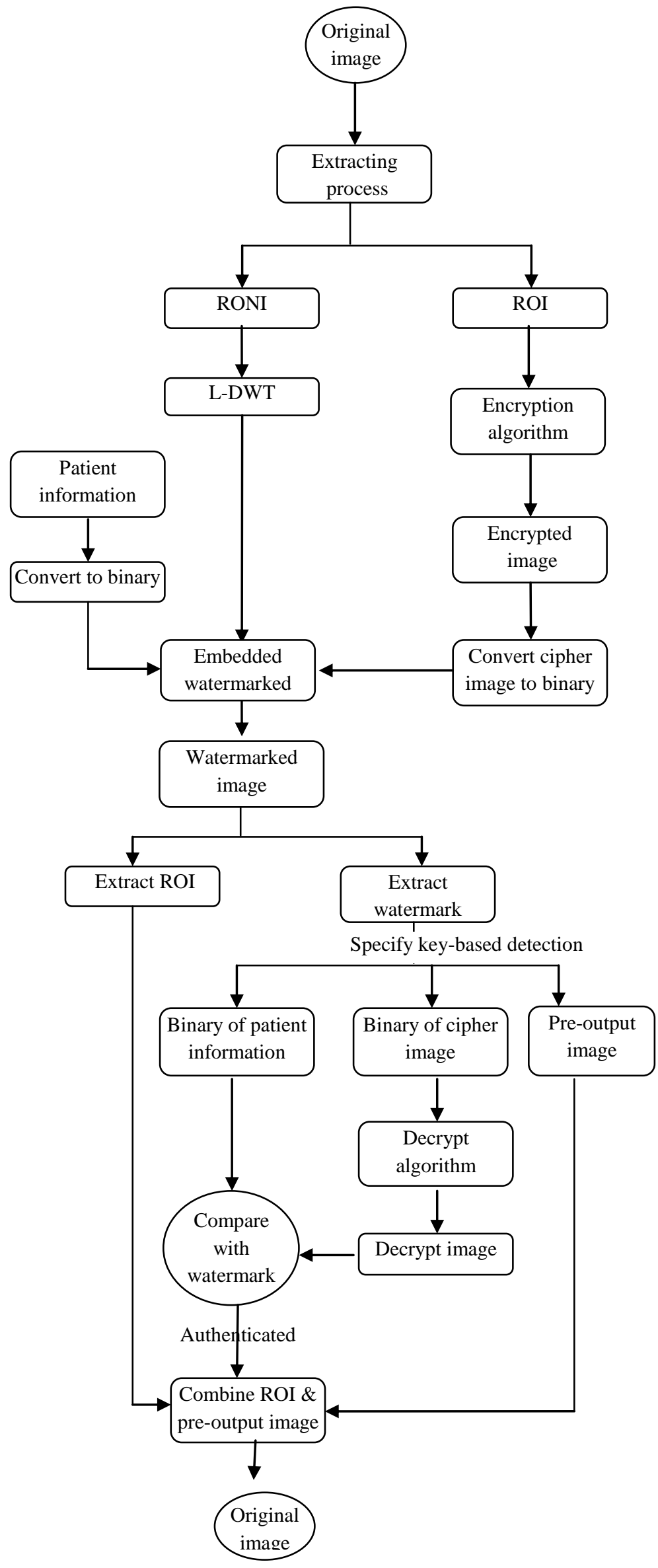

Fig (2) Summary of process 


\subsection{Extract ROI From Medical Image:}

This ROI usually is determined on the basis of pixel intensity values or user-determined areas (by drawing and subsequent masking). The process of separating objects of interest from uninteresting objects is called segmentation (Sonika C. Rathi et al., 2012).

The goal of segmentation is divide entire medical image in to sub regions. However segmentation is semiautomatic procedure and it needs to define a seed point in an image. Therefore, the algorithm, which gives perfect result for one type of medical image, may not even work for another. So we using graphical user interface (GUI) (Sonika C. Rathi et al., 2012).for defining the borders of ROI in all type of medical images such as CT scan, MRI, X-Ray, Ultrasound,etc.) that can simplify the phase of segmentation. Ymax.

In GUI application can select ROI and output pixel values of selected ROI region Xmin, Xmax, Ymin,

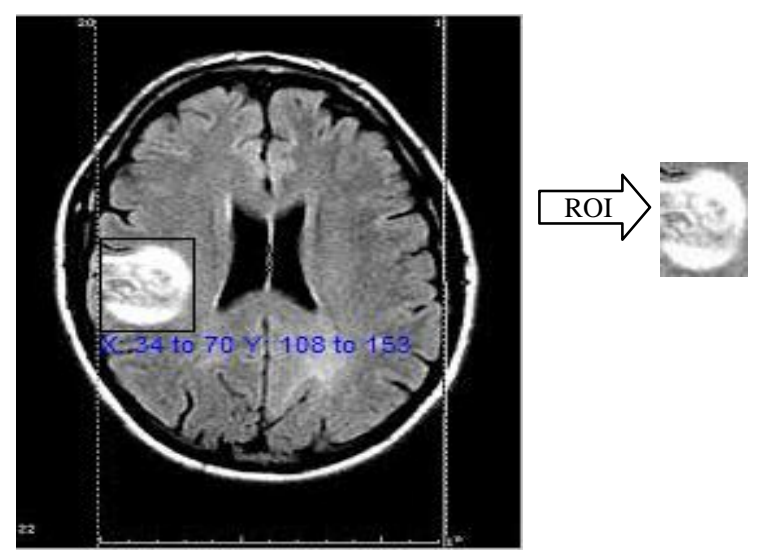

Fig. 3: Extract ROI with $X_{\min }=34, X_{\max }=70, Y_{\min }=108, Y_{\max }=153$

\subsection{Encrypt ROI using logistic map:}

Encrypt a sequence of bytes which is the 1D transformed version of the 2D original image. So we used independent logistic map of eq. (1) to give equation (2) is used to exchange pixel positions (Erdem Yavuza, Rifat Yazıcia et al., 2015),( Michael François, Thomas Grosges et al.,2012) (Musheer, Tanvir., 2012).

$$
x_{j+1}=\mu_{1} x_{j}\left(1-x_{j}\right)
$$

Where $\mathrm{j}>=0, \mathrm{x}_{0}$ initial seeds $\left.\epsilon\right] 0,1[$ then we follow the steps to encryption:

1- Convert ROI of size $\mathrm{MxN}$ to one array $\mathrm{Z}^{0}$ of size $8 x M x N$ in case color image $\mathrm{Z}^{0}=3 \times 8 \times \mathrm{MxN}$.

2- Specify iteration of logistic map [4] $r=1,2, \ldots, \mathrm{R}$ where $\mathrm{R}$ no. of round.

3- Specify initial condition:

- Used sequence of initial seeds are keys of encryption $\mathrm{x}_{0}^{1}, \mathrm{x}_{0}^{2}, x_{0}^{3} \ldots, x_{0}^{R}$

- $\alpha=10^{\mathrm{d}}$ is constant to scale $x_{j+1}^{r}$ and compute $\mathrm{d}=$ floor $\left[\log _{10} \mathrm{Z}\right]+3$

- $\sigma^{r}$ value of secret key according round

- $\quad \mathrm{r}=1 \& \mathrm{Z}^{\mathrm{r}}=\mathrm{Z}^{0}$ where $\mathrm{Z}^{\mathrm{r}}$ image array at round $\mathrm{r}, \mu_{1}=\mu_{2}=3.9999$

- $\mathrm{X}=x_{0}^{r}, \mathrm{Y}=y_{0}^{r}$

4- We handle the array $\mathrm{Z}$ consecutive using loop $\mathrm{i}=0$ to $\mathrm{i}=\mathrm{Z}-2$ then a new position $\mathrm{j}$ is computed by using the chaotic function equation (3):

$$
\mathrm{j}=\mathrm{i}+1+\left[\text { floor }\left[\alpha \mathrm{X}_{\mathrm{i}+1}\right] \bmod \mathrm{H}\right]
$$

Where $\mathrm{H}$ is initialize $\mathrm{Z}$-i i.e it decrease every iteration by 1 because modular operation select value of $\alpha \mathrm{X}_{\mathrm{i}+1}>\mathrm{H}$. And $\mathrm{i}<\mathrm{j}<\mathrm{Z}$

5- The elements of $Z^{r}=Z^{0}$ are transformed as loop: 


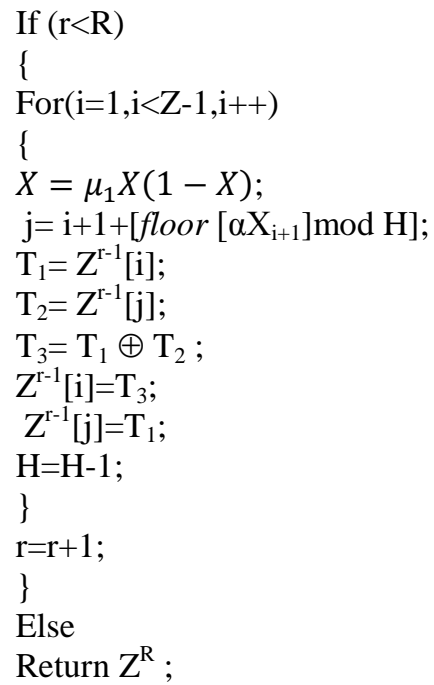

Where $\oplus$ XOR operation.

\subsection{Decrypt ROI using logistic map:}

We inverse the procedures of encryption to obtain the original ROI as follow (Erdem Yavuza, Rifat Yazicia al., 2015),( Michael François, Thomas Grosges et al.,2012):

\section{1- Input :}

- $\mathrm{Z}^{\mathrm{R}}, \mathrm{Z}, \mathrm{d}=$ floor $\left[\log _{10} \mathrm{Z}\right]+3, \mathrm{X}^{0} \ldots ., \mathrm{R}$

2- If $(r>0)$

\{

For $(\mathrm{i}=1, \mathrm{i}<\mathrm{Z}-1, \mathrm{i}++)$

\{

$X=\mu_{1} X(1-X)$;

$\mathrm{j}=\mathrm{i}+1+\left[\right.$ floor $\left.\left[\alpha \mathrm{X}_{\mathrm{i}+1}\right] \bmod \mathrm{H}\right]$;

$\mathrm{F}[\mathrm{i}]=\mathrm{j}$;

$\mathrm{H}=\mathrm{H}-1$;

\}

$\mathrm{j}=\mathrm{Z}-3$;

For $(j=Z-3 ; j \geq 0 ; j--)$

\{

$\mathrm{i}=\mathrm{F}[\mathrm{j}]$;

$\mathrm{T}_{1}=\mathrm{Z}^{\mathrm{R}}[\mathrm{j}]$;

$\mathrm{T}_{2}=\mathrm{Z}^{\mathrm{R}}[\mathrm{i}]$

$\mathrm{T}_{3}=\mathrm{T}_{1} \oplus \mathrm{T}_{2}$

$\mathrm{Z}^{\mathrm{R}}[\mathrm{j}]=\mathrm{T}_{2}$;

$\mathrm{Z}^{\mathrm{R}}[\mathrm{i}]=\mathrm{T}_{3}$;

\}

$\mathrm{r}=\mathrm{r}-1$;

\}

Else Return $\mathrm{Z}^{0}$

We apply the encryption and decryption algorithms on ROI image as shown in Fig (4) with initial condition: binary length of ROI image $38 \times 48 \quad \mathrm{Z}=14592$ bit and number of decimal digits $\mathrm{d}=$ floor $\left[\log _{10} \mathrm{Z}\right]+3=7$ and round number value 17 calculate according to equation (4). Then we convert the encryption image to binary to embed it in RONI as watermarks in addition to binary of patient information's as shown in next section . 


$$
R=\text { floor }\left[\log _{2}\left(\frac{\ln \left(\varepsilon_{2}\right)}{\ln \left(g_{0}\right.}\right)\right.
$$

Where $\varepsilon_{2}=0.005$ and $g_{0}=\frac{Z-1}{z}$

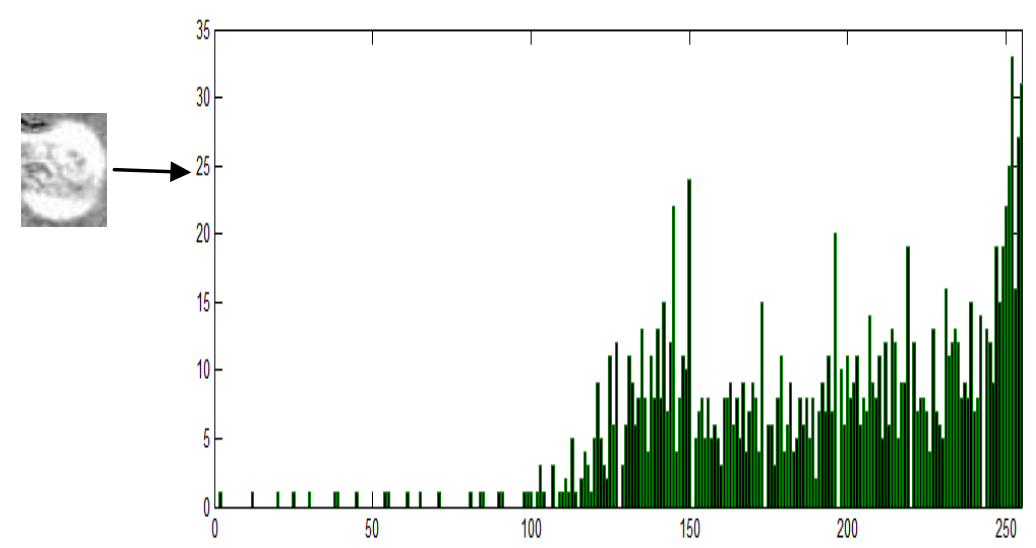

(a)

(b)

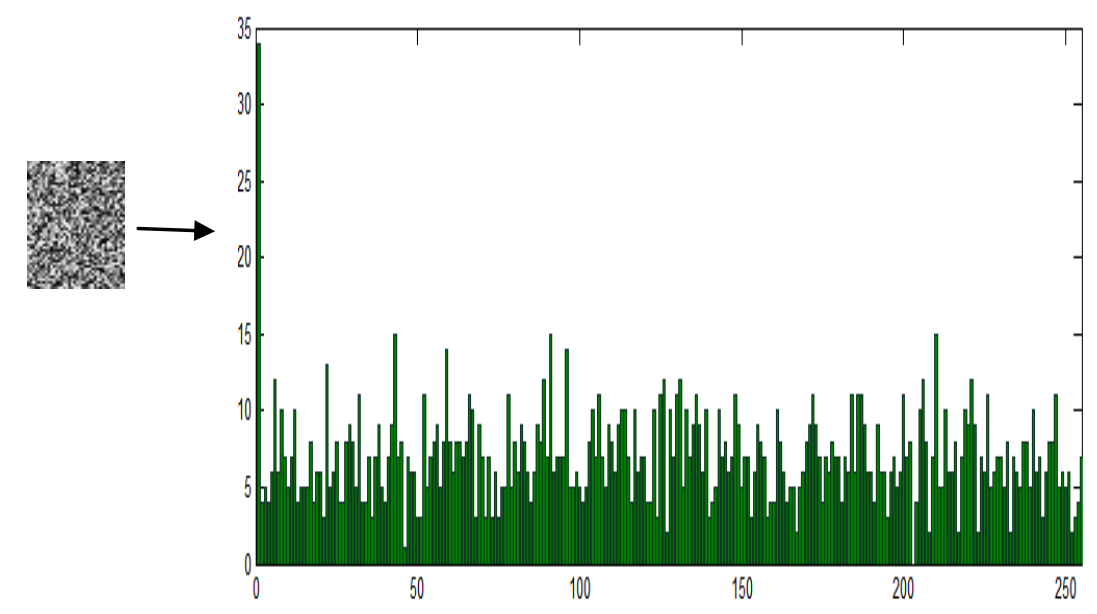

(c)

(d)

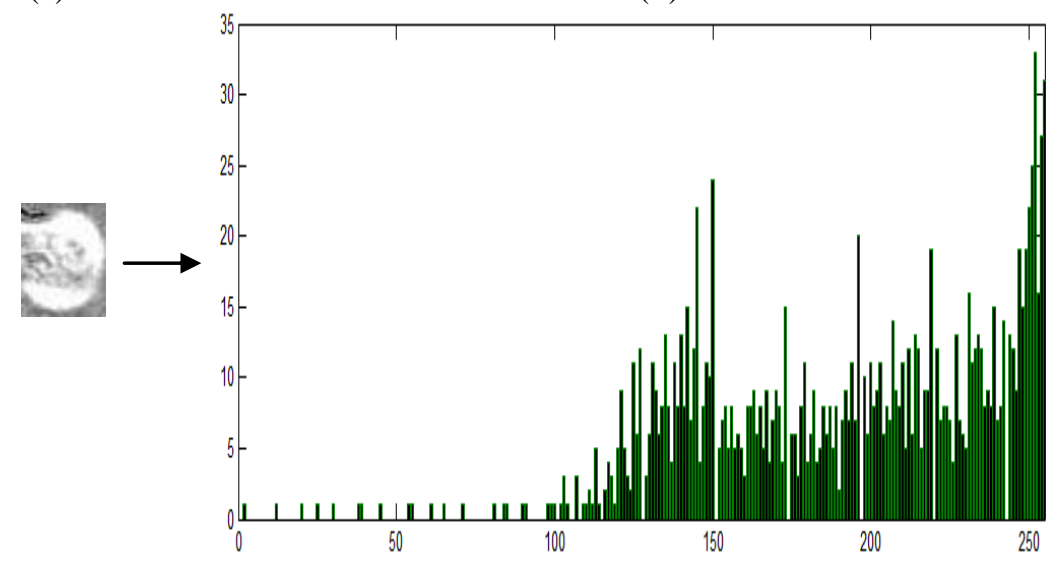

(e)

(f)

Fig. 4: (a) ROI as selected (b) histogram of ROI (c) Encrypted ROI (d) Histogram of Encrypted ROI (e) Decrypted ROI (f) Histogram of Decrypted ROI

\subsection{Embedding watermarks process:}

Watermark method according to domain (Jasni Mohamad Zain $†$ and Malcolm Clarke, 2007) is divided into two domains. One of them is spatial domain watermark depend on manipulates image pixels and it is simple to implement. But it is weak for some attacks such as the least significant bit (LSB). Second transform domain 
watermark depend on transform coefficients of cover image such as Discrete Wavelet Transform (DWT), Discrete Fourier Transform (DFT) and Discrete Cosine Transform (DCT)...etc.

In this paper we proposed to use 2D lifting discrete wavelet transform (L-DWT) (M. Santhosh, 2B. Stephen et al., 2013) as shown in fig (5) instead of 2D discrete wavelet transform (DWT) because it maintain the structural index and provides a better performance than the DWT (Siau-Chuin Liew et al.,2010) method. The lifting based DWT consists of three stages i.e. split, predict and update.

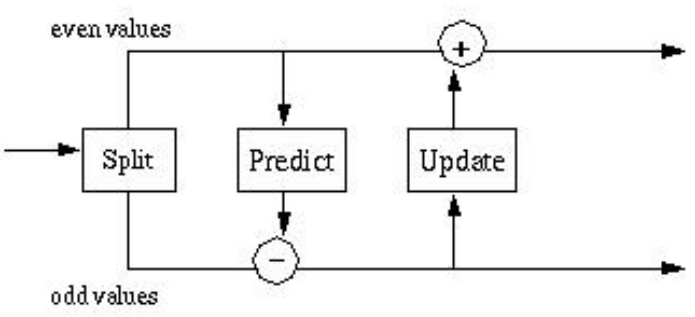

Fig. 5: scheme of lifting wavelet transform

In split stage the input signal $x[n]$ get divided into two subsets i.e. even set $e[n]$ and odd set o[n].

In predict step uses a function that approximates the data set. The difference between the approximation and the actual data replaces the odd elements of the data set (M. Santhosh, 2B. Stephen al., 2013) The even elements are left unchanged and become the input for the next step in the transform. The predict step, where the odd value is predicted from the even value is described by the equation (5).

$$
\operatorname{odd}_{\mathrm{j}+1, \mathrm{i}}=\operatorname{odd}_{\mathrm{j}, \mathrm{i}}-\mathrm{P}\left(\operatorname{even}_{\mathrm{j}, \mathrm{i}}\right)
$$

The update step lifts the even sequence values using the linear combination of the predicted odd sequence values so that the basic properties of the original sequence is preserved.

In this paper the multiple watermarks embedding technique is used based on the quantization of selected coefficients. We applying 4-level haar wavelet transform ( Hêmin Golpîra, Habibollah Danyali,2009) to decompose the RONI of medical image. And it gives the output as coefficients, these coefficients denominators are powers of 2 . The multiple of $2^{\wedge} \mathbf{i}$ ( $\mathrm{i}$ is level decomposition) number adding or subtracting to the produced coefficient value, assures that the inverse DWT provide integer pixel values. Wavelet transform provides the coefficients which are real numbers. By applying the quantization function it assigns the binary number to every coefficient. This quantization function is defined as equation (6) (Sonika C. Rathi et al.,2012).

$$
Q(s)= \begin{cases}0 & \text { if }\left\lfloor\frac{s-w}{\Delta}\right\rfloor \text { is even } \\ 1 & \text { if }\left\lfloor\frac{s-w}{\Delta}\right\rfloor \text { is odd }\end{cases}
$$

Where $\Delta$ the quantization parameter, is a positive real number. $\Delta$ is defined as $\Delta=2^{\wedge} i$. and $\mathrm{w}$ is a userdefined offset for increased security, $\mathrm{s}$ is frequency coefficient produced by haar wavelet transform.

We propose to embedding multiple watermarks one for patient information and second for cipher ROI of medical image. We follow the steps to achieve the embedding process (Sonika C. Rathi et al.,2012).

1) We convert patient information and cipher ROI to binary.

2) Apply 4-level Haar-lifting wavelet transform to RONI of medical image. This results in four decomposed sub images L4, H4, V4, and D4 for each image. the horizontal, vertical, and diagonal details at each of the four decomposition levels.

3) At each decomposition level the watermark bit wi is embedded into the key determined coefficient $\mathrm{s}$, which is output applying wavelet transform according to condition:

If $\mathrm{Q}(\mathrm{s})=$ wi, the coefficient is not modified, Otherwise, the coefficient is modified so that $\mathrm{Q}(\mathrm{s})=$ wi, using the following equation (7):

$$
s= \begin{cases}s+\Delta, & s \leq 0 \\ s-\Delta, & s>0\end{cases}
$$

4) The watermarked image is produced by performing the corresponding four level inverse wavelet transform.

5) The watermarked image that will be sent through hospital system is combined the ROI with the watermarks that obtain from step5. 


\subsection{Extraction watermarks process:} 2014):

We inverse the procedures of embedding to extract watermark as following steps (Neha Solanki et al.,

1) Extract the ROI from the received watermarked image depend on Xmax, Xmin, Ymin and Ymax parameter provided with watermarked image.

2) Apply the 4-level Haar- lifting wavelet transform to RONI, which results in a image approximation at 4-level and sequence of images horizontal, vertical, and diagonal details at each of 4-leveldecomposition.

3) Specify location of watermark by key-based detection.

4) Extract the watermarks by applying quantization function equation (6) which obtain the original coefficient.

5) Convert the extracted binary watermark to text watermark and cipher ROI of medical image.

6) Decrypt cipher ROI

7) Compare ROI output from step 6 to ROI output from 1 authenticate image.

8) The output image from inverse 4-level haar wavelet transform is combining with ROI to obtain original medical image.

\section{Experimental results:}

The proposed methodology has been simulated in MATLAB 7.12.0(R2011a) using different size of medical images such as magnetic resonance imaging (MRI), computed tomography (CT) scan, X-ray angiography, Ultrasound image,,.. etc.

In Fig (6) (a) original CT scan image for brain tumor image and its histogram; then embedded patient information and cipher ROI as multiple watermarks in RONI as shown in fig(6) (b) watermarked image and its histogram.

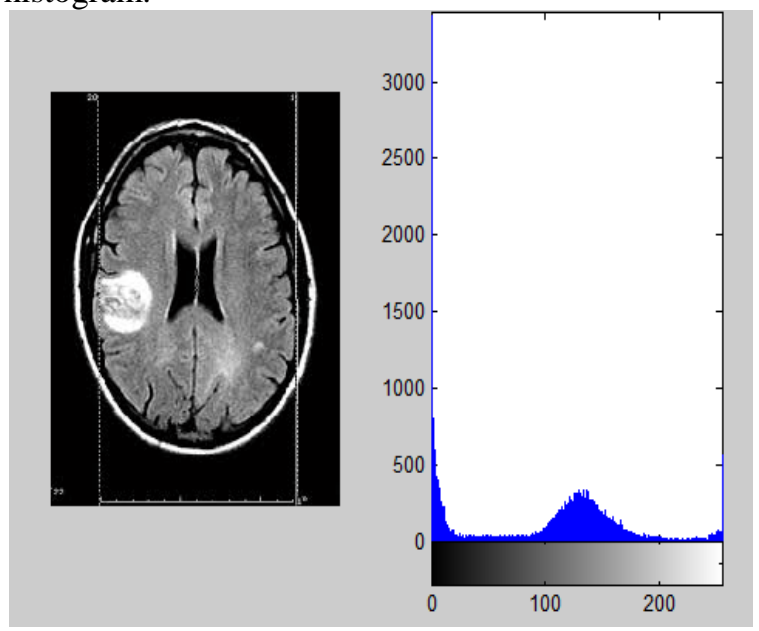

(a)

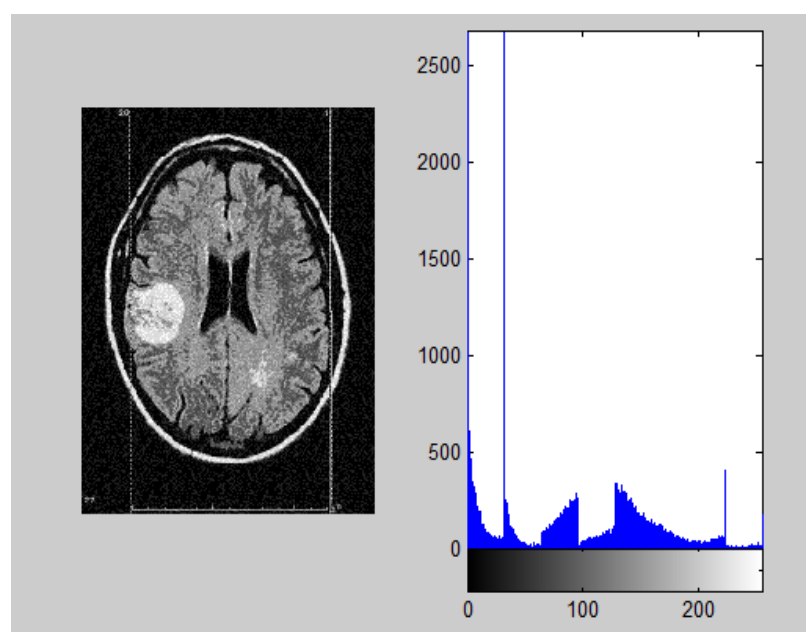

(b)

Fig. 6: (a) original image \& histogram (b) watermarked image \& histogram

In Fig (7) apply proposed system on magnetic resonance imaging (MRI) first select ROI $35 \times 36$ then encrypt with initial condition: binary length $Z=8 \times 35 \times 36=10080$ bit, decimal digits $\mathrm{d}=$ floor $\left[\log _{10} \mathrm{Z}\right]+3=7$ and round number value 15 calculate according to equation (4) with $\varepsilon_{2}=0.005$. Then convert cipher ROI to binary and embedded it with patient information into RONI.

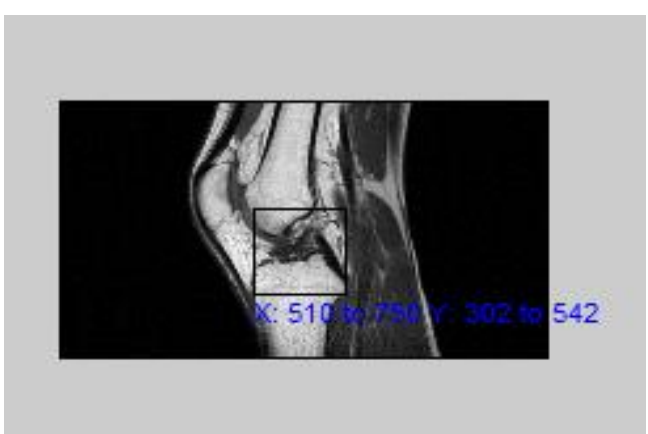

(a)

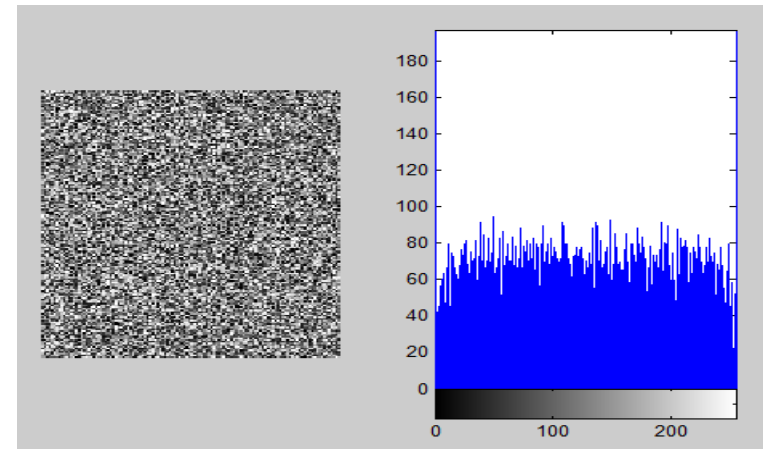

(b) 


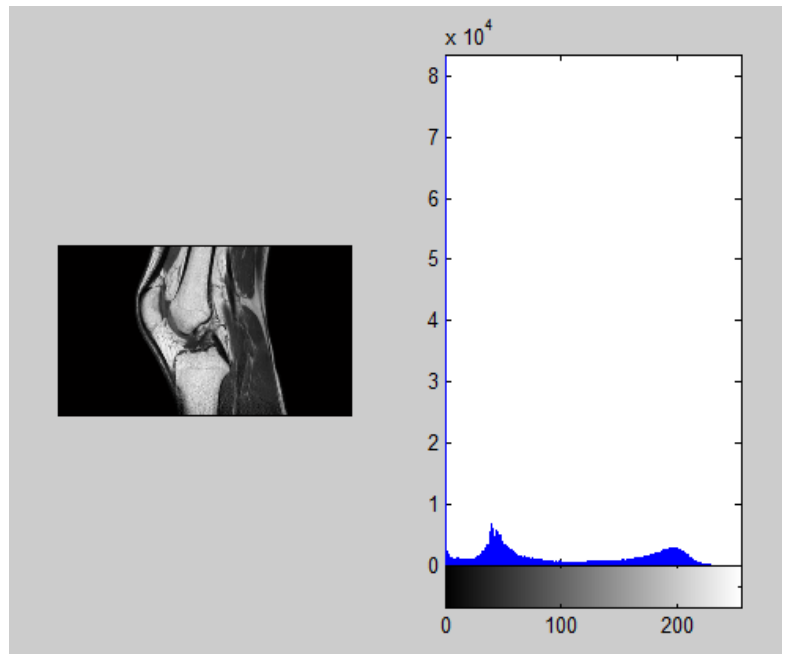

(c)

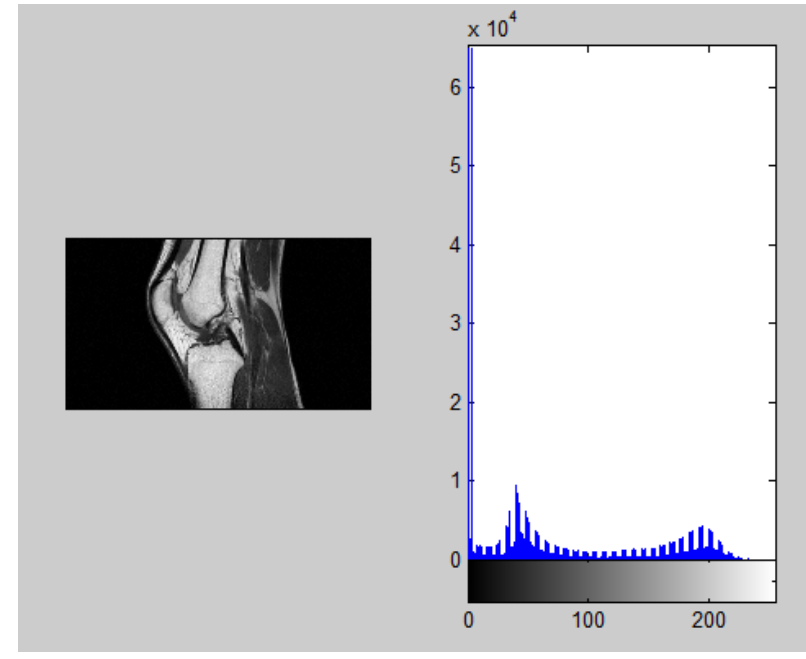

(d)

Fig. 7: (a) select ROI original image (b)Encrypt ROI \&histogram (c) original image \& histogram (d) watermarked image \& histogram

We apply proposed on X-ray image as show in Fig (8) first select ROI as (a) of size $165 \times 223$ then encrypt with initial condition: binary length $Z=8 \times 165 \times 223=294360$ bit, decimal digits $\mathrm{d}=$ floor $\left[\log _{10} \mathrm{Z}\right]+3=8$ and round number value 20 calculate according to eq (4) with $\varepsilon_{2}=0.005$. Then convert encrypted ROI to binary and embedded it with patient information into RONI.

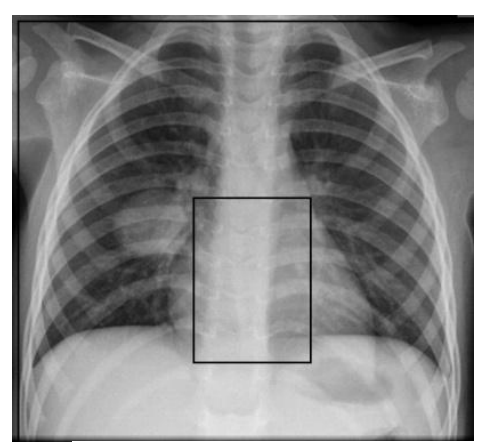

(a)

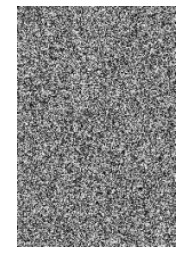

(b)

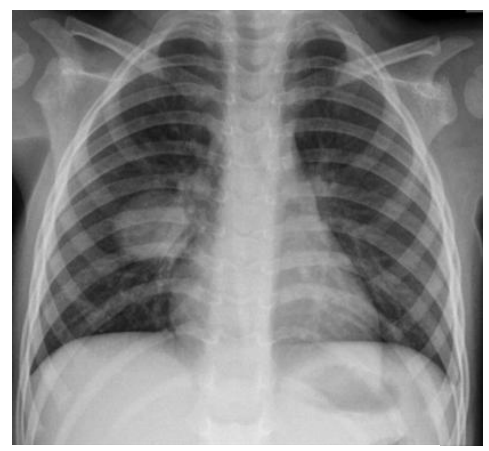

(c)

Fig. 8: (a) select ROI original image (b) Encrypt ROI (c) watermarked image

Table 1 illustrate the reliability and quality of the watermarked image, the performance of watermarking is calculated, which measured in terms of perceptibility. There are two method of calculating the performance measure:

1- Mean Square Error (MSE): It is simplest function to measure the perceptual distance between authenticated image after imbedding watermark and original image can be defined as equation (8):

$$
M S E=\frac{1}{n} \sum_{i}^{n}\left(I^{\prime}-I\right)^{2}
$$

2- The Peak Signal to Noise Ratio (PSNR) ( Siau-Chuin Liew et al.,2010) in dB compare between authenticated image after imbedding watermarks and original image is define as equation (9) :

$$
P S N R=10 * \log _{10}\left(\frac{M A X_{I}^{2}}{M S E}\right)
$$


Table 1: MSN \& PSNR measures for CT, MRI and X ray image using proposed algorithm

\begin{tabular}{|c|c|c|c|c|c|c|c|c|}
\hline \multirow{2}{*}{$\begin{array}{c}\text { No. of } \\
\text { image }\end{array}$} & \multirow{2}{*}{$\begin{array}{c}\text { Image } \\
\text { type }\end{array}$} & \multirow{2}{*}{ Size } & \multicolumn{2}{|c|}{$\begin{array}{c}\text { Michael, Thomas } \\
\text { al.(2012) }\end{array}$} & \multicolumn{2}{c|}{$\begin{array}{c}\text { Yavuz, Erdem } \\
\text { al.(2015) }\end{array}$} & \multicolumn{2}{c|}{ Proposed } \\
\cline { 4 - 9 } & & MSE & PSNR & MSE & PSNR & MSE & PSNR \\
\hline $\mathbf{1 0}$ & $\begin{array}{c}\text { CT image } \\
\text { fig (6) }\end{array}$ & $\mathbf{1 9 5 x 2 5 9}$ & 0.382 & 52.901 & 0.324 & 52.231 & 0.4075 & 52.0299 \\
\hline $\mathbf{1 5}$ & $\begin{array}{c}\text { MRI } \\
\text { fig (7) }\end{array}$ & $\mathbf{1 2 8 0 \times 7 2 0}$ & 0.325 & 52.106 & 0.436 & 51.465 & 0.3677 & 52.4763 \\
\hline $\mathbf{6}$ & $\begin{array}{l}\text { X-ray } \\
\text { fig(8) }\end{array}$ & $\mathbf{1 2 1 5 x 1 1 2 5}$ & 0.427 & 51.367 & 0.527 & 50.137 & 0.4992 & 51.1484 \\
\hline \multicolumn{2}{|c|}{ Average } & & 0.378 & 52.124 & 0.429 & 51.277 & 0.4248 & 51.8848 \\
\hline
\end{tabular}

From Table 1 show the comparison between the proposed algorithm and previous algorithm such as Michael, Thomas et al. (2012) and Yavuz, Erdem et al.(2015). Average of MSE equal about 0.378 for Michael, Thomas et al. (2012), 0.429 for Yavuz, Erdem et al. (2015) and 0.4248 for proposed ; it indicates that the MSE has very low consistent value for first algorithm because they apply chaotic algorithm only so this comparison show that the proposed algorithm more efficient and give the best result with MSE 0.4248 this mean that the embedded watermark does not affect the quality of the original images. The average of PSNR equal about $51.8848 \mathrm{~dB}$.

\section{Conclusion:}

Due to the importance of securing patient's information and medical image while transfer we proposed system based on multilayer security that combine chaos map and watermark. Frist used to select ROI from medical image that will be applicable for all types of medical images. Then separated ROI can be stored with xmin, xmax, ymin, and ymax value. In our scheme encrypt ROI then concatenate with patient's information to produce watermarks that embedded in RONI of medical image. The experimental results prove that the proposed system can provide system authentication, image integrity and patient information confidentiality with high efficiency comparison with other algorithm using vary digital images such as: nuclear medicine, ultrasound, X-ray, computed radiography (CR) computed tomography (CT)...etc. . And an average of MSE for proposed about 0.424 mean that proposed embedded watermark does not affect the quality of the original images and in the same time securing patient's information.

\section{REFERENCES}

Erdem Yavuza, Rifat Yazicia et al., 2015. "A chaos-based image encryption algorithm with simple logical functions". Computer and Electrical Engineering, pp: 1-13.

Hao, Z., W. Xing-yuan, W. Si-wei, G. Kang, L. Xiao-hui, 2017. “Application of coupled map lattice with parameter q in image encryption”. Optics and Lasers in Engineering, 88: 65-74.

Hêmin Golpîra, Habibollah Danyali, 2009. "Reversible blind watermarking for medical images based on wavelet histogram shifting". IEEE.

Hussain, I., T. Shah, 2013. "Application of S-box and chaotic map for image encryption". Math Comput Modell., 57: 2576-9.

Jasni Mohamad Zain $\dagger$ and Malcolm Clarke, 2007. "Reversible Region of Non-Interest (RONI) Watermarking for Authentication of DICOM Images". IJCSNS International Journal of Computer Science and Network Security, 7: 9.

Santhosh, M., 2B. Stephen et al., 2013.” Image Decomposition and Compression by using Lifting Scheme". International Journal of Engineering and Innovative Technology (IJEIT) 3: 2.

Mamta Jain, Anil Kumar, 2017.” Improved diagonal queue medical image steganography using Chaos theory, LFSR, and Rabin cryptosystem”. Brain Informatics, 4: 95-106.

MATLAB. The language of technical computing. MathWorks Company, 2017. http://www.mathworks.com/products/matlab/ index.html .

Michael François, Thomas Grosges et al., 2012. "Image Encryption Algorithm Based on a Chaotic Iterative Process". Applied Mathematics, 3: 1910-1920. 
Musheer, Tanvir, 2012. " A framework to protect patient digital medical imagery for secure telediagnosis".procedia engineering 381055-1066.

Neha Solanki, et al., 2014. "ROI Based Medical Image Watermarking with Zero Distortion and Enhanced Security". I.J. Modern Education and Computer Science., 10: 40-48.

Pan, W., G. Coatrieux, N. Cuppens-boulahia, "Digital Signature and Lossless Watermarking. Imaging". Springer, pp: 153-162.

Quist, A., N. Laurent et al., 2015. " A cryptographic technique for security of medical images in health information systrms". Procedia Computer Science., 58: 538-543.

Siau-Chuin Liew et al., 2010. "Reversible Medical Image Watermarking For Tamper Detection And Recovery".IEEE 978-1-4244-5540.

Sonika, C., Rathi et al., 2012." Medical Images Authentication Through Watermarking Preserving ROI”. Health Informatics, 1: 1.

Wang, Y., Wong K-wo, X. Liao, T. Xiang, G. Chen Chaos, Solitons and Fractals, 2009. "A chaos-based image encryption algorithm with variable control parameters". Chaos, Solitons and Fractals, 41(4): 1773-1783. 